\title{
Energy Loss of Radio Pulsars
}

\author{
V.S. Beskin
}

Lebedev Physical Institute, Leninskii prosp. 53, Moscow, 117924, Russia

R.R. Rafikov

Princeton University Observatory, Princeton, NJ 08544, USA

\begin{abstract}
Two-fluid effects on the radial 2D outflow of relativistic electron-positron plasma are considered. It is shown that when the longitudinal electric current is smaller than the Goldreich-Julian one, almost all electromagnetic energy is transformed into the energy of particles in the narrow boundary layer near the light surface $|\mathbf{E}|=|\mathbf{B}|$.
\end{abstract}

\section{Introduction}

Starting from Pacini (1967), magnetodipole radiation

$$
W_{\mathrm{md}} \approx \frac{1}{6} \frac{B_{0}^{2} \Omega^{4} R^{6}}{c^{3}} \sin ^{2} \chi
$$

was considered as a main mechanism of the braking of radio pulsars. Indeed, it can explain the energy losses for reasonable magnetic fields $B_{0} \sim 10^{12} \mathrm{G}$ and main statistical characteristics of radio pulsars. Nevertheless, this model met some problems, e.g.

- braking index $n_{\mathrm{br}}=\Omega \ddot{\Omega} / \dot{\Omega}^{2}$ for the magnetodipole mechanism is $n_{\mathrm{br}}=$ $3+2 \cot ^{2} \chi>3$, but observations give $n<3$,

- the heating of the companion in close binaries (see e.g. Djorgovski \& Evans 1988) cannot be explained because magnetodipole wave cannot heat the companion efficiently.

On the other hand, in the presence of the longitudinal electric current $I$ flowing in the magnetosphere the efficiency of the electric current loss mechanism (Goldreich \& Julian 1969)

$$
W_{\text {cur }} \sim \frac{B_{0}^{2} \Omega^{4} R^{6}}{c^{3}} \frac{I}{I_{\mathrm{GJ}}}
$$

is close to the magnetodipole one. This mechanism could explain not only the statistical characteristics of radio pulsars and the energy loss itself, but the small enough braking index $n_{\mathrm{br}}=1.93+1.5 \tan ^{2} \chi$ as well (Beskin, Gurevich, \& Istomin 1993). Moreover, exact solution for the oblique rotator with zero 
longitudinal electric current demonstrates that plasma screens fully the magnetodipole radiation of the central star (Beskin, Gurevich, \& Istomin 1983; Mestel, Panagi, \& Shibata 1999).

Further, it was shown that if the longitudinal electric current is determined by the critical conditions at the singular surfaces, its value is to be of the order of Goldreich-Julian one $I \geq I_{\mathrm{GJ}}$, with the light surface $|\mathbf{E}|=|\mathbf{B}|$ located at infinity. Unfortunately, in this case the transformation of the electromagnetic energy into the energy of particles is ineffective (Tomimatsu 1994; Bogovalov 1997; Beskin, Kuznetsova, \& Rafikov 1998; Chiueh, Li, \& Begelman 1998). It means that within this model it is impossible to explain the heating of companion in close binaries containing radio pulsars. On the other hand, if there are some physical restrictions of the longitudinal current flowing in the magnetosphere, so that $I<I_{\mathrm{GJ}}$, then the effective particle acceleration can take place in the vicinity of the light surface $|\mathbf{E}|=|\mathbf{B}|$ located at a finite distance from the neutron star.

An example of the restriction of the longitudinal electric field for axisymmetric case in the force-free approximation was already discussed within the Ruderman-Sutherland model of the internal gap (Beskin, Gurevich, \& Istomin 1983) and was recently reconsidered by Beskin \& Malyshkin (1998). It was shown that there is a connection between the electric current $i_{0}=I / I_{\mathrm{G}} \mathrm{J}$ flowing in the magnetosphere and the potential drop in the internal gap $\beta_{0}=\psi / \psi_{\max }$. It appears as a result of the interaction between regions with open and closed magnetic field lines. In the last paper it was additionally taken into account that the matching condition along the separatrix (Okamoto 1974; Lyubarskii 1990)

$$
\left\{B^{2}-E^{2}\right\}=0
$$

is to be valid.

As a result, it was found that in general case the zero point of the magnetic field is located inside the light cylinder, but the minimum of the total energy corresponds to the solution when the zero point is exactly at the light cylinder. For this reason, the relation between the electric current and the potential drop

$$
\beta_{0}\left(i_{0}\right)=1-\left(1-\frac{i_{0}^{2}}{i_{\max }^{2}}\right)^{1 / 2}
$$

$\left(i_{\max }=1.58\right)$, which was predicted by Beskin, Gurevich, \& Istomin (1983), remains true.

It is necessary to stress that the longitudinal current might be restricted in the Arons (1983) model as well. Indeed, within this model the electric current $I \approx I_{\mathrm{GJ}}$ is determined by the internal gap structure. In general case this current does not correspond to the critical condition at the fast magnetosonic surface. In particular, it may be smaller than the critical current (of course, the detailed consideration is necessary to check this statement).

Thus, the light surface can indeed be located at a finite distance from the light cylinder. Since the frozen-in condition $\mathbf{E}+\mathbf{v} \times \mathbf{B} / c=0$ can not be valid there, particles have the possibility to move in the direction perpendicular to magnetic field lines. It is this motion along the electric field that results in effective acceleration of plasma flowing out from the pulsar magnetosphere. 


\section{Particle Acceleration near the Light Surface}

In this proceeding we consider 2D generalization of two-fluid equations (see Beskin et al. 1983) describing the particle acceleration in the vicinity of the light surface. It gives the possibility to include the disturbances of the poloidal magnetic field and electric potential into consideration. As a zero order approximation we consider Michel (1973) one-fluid monopole outflow

$$
\begin{aligned}
B_{r}^{0} & =B_{\mathrm{s}} \frac{R_{\mathrm{s}}^{2}}{r^{2}}, \\
B_{\varphi}^{0} & =E_{\theta}^{0}=-B_{\mathrm{s}} \frac{R_{\mathrm{s}} \Omega}{c} \frac{R_{\mathrm{s}}}{r} \sin \theta, \\
v_{\theta} & =0 .
\end{aligned}
$$

We also assume that the longitudinal current is small enough to pass smoothly through the fast magnetosonic surface

$$
I=(1-h) I_{\mathrm{GJ}} \sin ^{2} \theta,
$$

where $h \ll 1$. In this case the light surface $|\mathbf{E}|=|\mathbf{B}|$ is located at a finite distance

$$
\varpi_{c}=\frac{R_{\mathrm{L}}}{(2 h)^{1 / 4}} .
$$

For our choice of the current $I(8)$ this surface has the form of a cylinder.

As a result, solving two-fluid equations of motion for electrons and positrons (for more details see Beskin \& Rafikov 2000), we found that for real physical parameters of the magnetosphere of radio pulsars $(\sigma \gg 1$ and $\lambda \gg 1)$ the onefluid MHD approximation remains valid in the whole region inside the light surface $|\mathrm{E}|=|\mathrm{B}|$. Here $\lambda=n / n_{\mathrm{G} . \mathrm{J}} \sim 10^{3}-10^{4}$ is the multiplication parameter, and

$$
\sigma=\frac{\Omega^{2} e B_{0} R^{3}}{4 \lambda m c^{4}} \approx 10^{4} B_{12} \lambda_{3}^{-1} P^{-2} \sim 10^{3}-10^{4},
$$

is the Michel (1969) magnetization parameter. On the other hand, in the boundary layer near the light surface where the effective particle acceleration takes place the solution has the following simple form for $\lambda l \ll 1$

$$
\begin{aligned}
\gamma^{ \pm} & =4 \sin ^{2} \theta \sigma(\lambda l)^{2}, \\
v_{\theta}^{ \pm} / c & =\mp 2 \sin \theta \lambda l, \\
\delta B_{\varphi} / B_{\varphi} & =-4 \sin \theta(\lambda l)^{2},
\end{aligned}
$$

where $l=\Delta \varpi / \varpi_{c}$.

As we can see, in the narrow layer $\Delta \varpi=\varpi_{c} / \lambda$ the particle energy increases up to the value $\sigma m_{e} c^{2}$ which corresponds to the full conversion of the electromagnetic energy into the energy of particles $W_{\text {part }} \sim W_{\text {tot }}$. For this reason it is not surprising that here $\delta B_{\varphi} / B_{\varphi} \approx 1$, which just means the vanishing of the toroidal magnetic field i.e. almost total closure of the longitudinal current. On the other hand, the variation of the electric potential remains small $\sim \lambda^{-1}$, to say nothing about the variation of the magnetic surfaces $\sim \lambda^{-2}$. These results coincide exactly with our previous evaluations allowing us to neglect variations of the electric potential and the poloidal magnetic structure in the $1 \mathrm{D}$ cylindrical case. 


\section{Conclusions}

Thus, on a simple example it was demonstrated that for real physical parameters of the magnetosphere of radio pulsars $(\sigma \gg 1$ and $\lambda \gg 1)$ in a realistic $2 \mathrm{D}$ case the main properties of the boundary layer near the light surface (effective energy transformation from electromagnetic field to particles, current closure in this region, smallness of the disturbance of electric potential and poloidal magnetic field) remain the same as in the $1 \mathrm{D}$ case considered previously.

Nevertheless, as in the cylindrical case, our approach does not allow to describe the particle motion outside the light surface. The point is that, for a complete conversion of electromagnetic energy into the energy of particles it is enough for them to pass only $\lambda^{-1}$ of the total potential drop between pulsar magnetosphere and infinity. It means that the electron-positron wind propagating to infinity has to pass the potential drop which is much larger than their energy. It is possible only in the presence of electromagnetic waves even in an axisymmetric magnetosphere which is stationary near the origin. Clearly, such a flow cannot be considered even within the two-fluid approximation. In our opinion, it is only a numerical consideration that can solve the problem completely and determine, in particular, the energy spectrum of particles and the structure of the pulsar wind. Unfortunately, up to now such numerical calculations are absent.

Acknowledgments. This work was supported by INTAS Grant 96-154.

\section{References}

Arons, J. 1983, ApJ, 266, 215

Beskin, V.S., Gurevich, A.V., \& Istomin, Ya.N. 1983, Soviet Phys. JETP, 58, 235

Beskin, V.S., Gurevich, A.V., \& Istomin, Ya.N. 1993, Physics of the Pulsar Magnetosphere, Cambridge Univ. Press, Cambridge

Beskin, V.S., \& Malyshkin, L.M. 1998, MNRAS, 298, 847

Beskin, V.S., Kuznetsova, I.V., \& Rafikov, R.R. 1998, MNRAS, 299, 341

Beskin, V.S., \& Rafikov, R.R. 2000, MNRAS, (in press)

Bogovalov, S.V. 1997, A\&A, 327, 662

Chiueh, T., Li, Z. -Y., \& Begelman, M. C. 1998, ApJ, 505, 835

Goldreich, P., \& Julian, W.H. 1969, ApJ, 160, 971

Djorgovski, S., \& Evans, C.R. 1988, ApJ, 335, L61

Lyubarskii, Yu. E. 1990, Soviet Astron. Lett., 18, 356

Mestel, L., Panagi, P., \& Shibata, S. 1999, MNRAS, 309, 388

Michel, F.C. 1969, ApJ, 158, 727

Michel, F.C. 1973, ApJ, 180, L133

Okamoto, I. 1974, MNRAS, 167, 457

Pacini, F. Nature, 1967, 221, 567

Tomimatsu, A. 1994, PASJ, 46, 123 